\title{
Optimization of Fuzzy c-means (FCM) Clustering in Cytology Image Segmentation Using the Gray Wolf Algorithm
}

Maryam Mohammdian-khoshnoud

Hamadan University of Medical Sciences

Ali Reza Soltanian ( $\nabla$ soltanian@umsha.ac.ir)

Hamadan University of Medical Sciences

Arash Dehghan

Hamadan University of Medical Sciences

Maryam Farhadian

Hamadan University of Medical Sciences

\section{Research Article}

Keywords: Computer-aided diagnosis, Breast cancer, Image segmentation, Machine learning, Gray wolf optimization, Fuzzy c-means, Optimization

Posted Date: September 7th, 2021

DOI: https://doi.org/10.21203/rs.3.rs-808322/v1

License: (c) (i) This work is licensed under a Creative Commons Attribution 4.0 International License. Read Full License

Version of Record: A version of this preprint was published at BMC Molecular and Cell Biology on February 15th, 2022. See the published version at https://doi.org/10.1186/s12860-022-00408-7. 
Optimization of fuzzy c-means (FCM) clustering in cytology image segmentation using the gray wolf algorithm

Maryam Mohammdian-khoshnoud ${ }^{1}$, Ali Reza Soltanian*2, Arash Dehghan ${ }^{3}$, Maryam Farhadian ${ }^{4}$

${ }^{1}$ Department of Biostatistics, School of Public Health and Research Center for Health Sciences, Hamadan University of Medical Sciences, Hamadan, Iran. Email address: info.amargiran@ gmail.com

${ }^{2}$ Modeling of Noncommunicable Diseases Research Center, Hamadan University of Medical Sciences, Hamadan, Iran. Email address: soltanian@umsha.ac.ir

${ }^{3}$ Department of Pathology, School of Medicine, Hamadan University of Medical Sciences, Hamadan, Iran. Email address: a.dehghan50@yahoo.com.

${ }^{4}$ Department of Biostatistics, School of Public Health and Research Center for Health Sciences, Hamadan University of Medical Sciences, Hamadan, Iran. Email address: maryam_farhadian80@yahoo.com

*(Corresponding author): Ali Reza Soltanian, soltanian@umsha.ac.ir 


\begin{abstract}
Background: Image segmentation is considered an important step in image processing. Fuzzy cmeans clustering is one of the common methods of image segmentation. However, this method suffers from drawbacks, such as sensitivity to initial values, entrapment in local optima, and the inability to distinguish objects with similar color intensity. This paper proposes the hybrid Fuzzy cmeans clustering and Gray wolf optimization for image segmentation to overcome the shortcomings of fuzzy c-means clustering. The Gray wolf optimization has a high exploration capability in finding the best solution to the problem, which prevents the entrapment of the algorithm in local optima. In this study, breast cytology images were used to validate the methods, and the results of the proposed method were compared to those of c-means clustering.
\end{abstract}

Results: FCMGWO has performed better than FCM in separating the nucleus from the other dark objects in the cell. The clustering was validated using Vpc, Vpe, Davies-Bouldin, and Calinski Harabasz criteria. The FCM and FCMGWO methods have a significant difference with respect to the $\mathrm{Vpc}$ and Vpe indices. However, there is no significant difference between the performances of the two clustering methods with respect to the Calinski-Harabasz and Davies-Bouldin indices. The results indicate the better efficacy of the proposed method.

Conclusions: The hybrid FCMGWO algorithm distinguishes the cells better in images with less detail than in images with high detail. However, FCM exhibits unacceptable performance in both low- and high-detail images.

Keywords: Computer-aided diagnosis, Breast cancer, Image segmentation, Machine learning, Gray wolf optimization, Fuzzy c-means, Optimization 


\section{Background}

Image segmentation is the division of an image into discrete regions such that the pixels inside each region have the highest similarity and those across different regions have the highest contrast(1). Threshold-based, edge-based, region-based, matching-based, clustering-based segmentation, segmentation based on fuzzy inference and generalized principal component analysis are image segmentation techniques(2). Each of these methods has advantages and disadvantages. Consequently, none of them can be considered a comprehensive image segmentation algorithm(2). Image segmentation can be considered a classification problem. Hence, machine-learning-based classification algorithms can be of great help in this area(3). Fuzzy c-means (FCM) clustering is one of the important unsupervised learning algorithms. It requires knowledge of the initial details of some of the parameters, such as the number of clusters and the position of the centroid of the clusters, and its performance depends on the input parameters. Some researchers have proposed various methods for estimating the number of clusters or cluster centroids $(4,5)$. Moreover, FCM is sensitive to noise and entrapment in local optima. Various metaheuristic methods have been used to optimize the objective function of the fuzzy algorithm in order to avoid entrapment in local optima. Also, FCM fails in distinguishing objects with similar color intensity in images on its own. To overcome the mentioned issues, the Gray wolf optimization(GWO) was used for optimization in this research(6). The combined use of FCM and GWO to find the optimal cluster centers improves the cluster performance. The main criterion for selecting the best algorithm for medical images is the accuracy of the algorithms. Reducing complexity is the next goal in medical image processing. Therefore, the present study aims to combine FCM with the GWO. Using this combination prevents entrapment in local optima and better optimizes the cluster centers obtained from FCM. In addition, the clustering 
will be more capable of distinguishing the nucleus from the cytoplasm and other dark-colored cell features in breast cancer cytology images.

\section{Results}

The performances of the FCMGWO and FCM in segmenting cytology images were compared. The performance of the algorithms was evaluated using $\mathrm{V}_{\mathrm{pc}}, \mathrm{V}_{\mathrm{pe}}, \mathrm{DB}$, and $\mathrm{CH}$ validation indices. The clustering result is acceptable when $\mathrm{V}_{\mathrm{pc}}$ and $\mathrm{CH}$ are maximum and $\mathrm{V}_{\mathrm{pe}}$ and $\mathrm{DB}$ are minimum (Fig. 2). A study of the indices presented in Fig. 2 reveals the superiority of FCMGWO over FCM with $\mathrm{V}_{\mathrm{pe}}$ and $\mathrm{V}_{\mathrm{pc}}$ criteria for all images. According to the $\mathrm{CH}$ index, FCMGWO is better than FCM for images 3 and 4, while FCM is better than FCMGWO for images 1 and 2. The image segmentation results can be seen in Fig. 1. In all the analyzed images, FCMGWO has performed better than FCM in separating the nucleus from the other dark objects in the cell. The points corresponding to the nucleus and other dark objects, such as cytoplasm and red blood cells, have been considered as one cluster by FCM. In FCMGWO, however, these points have been designated as the nucleus, and the other objects have been distinguished as two clusters.

Figure 1. Cytology images segmented using FCM and FCMGWO

Figure 2. Validation indices for comparing FCM and FCMGWO

The paired t-test was used to compare the significance between the indices. The normality of the $V_{p c}$, $\mathrm{V}_{\mathrm{pe}}, \mathrm{DB}$, and $\mathrm{CH}$ indices was examined using the Shapiro-Wilk test (p-value $>0.05$ ). Then, the paired t-test was used to investigate the significance of the differences in the indices (Table 1). The FCM and FCMGWO methods have a significant difference with respect to the $\mathrm{V}_{\mathrm{pc}}$ and $\mathrm{V}_{\mathrm{pe}}$ indices. However, there is no significant difference between the performances of the two clustering methods with respect to the $\mathrm{DB}$ and $\mathrm{CH}$ indices. 
Table 1. Paired t-test results indicating the difference between the performance of the indices

\section{Discussion}

The segmentation of images containing objects with similar color intensity is a challenge in image processing. It is difficult to distinguish the cell nucleus from other cell components, such as red blood cells and plasma, in cytology images due to color similarities. The current study proposed the FCMGWO method for this purpose. This technique was compared to the FCM and validated for breast cytology images.

The results indicate that FCM is incapable of identifying the cell nucleus. FCM considers the nucleus and other dark objects in the cell as one cluster and cannot distinguish between them. However, the combined FCMGWO method performs better than FCM in distinguishing the cell nuclei. This better discernment can be due to the search process of the GWO, which optimizes the cluster centers obtained from FCM clustering. This optimization can improve the performance of FCM and overcome some of its shortcomings owing to its high exploration capability and the good agreement between the exploration and exploitation in GWO.

The improvement in $\mathrm{V}_{\mathrm{pc}}$ and $\mathrm{V}_{\mathrm{pe}}$ using FCMGWO is more statistically significant compared to FCM. However, no significant difference was observed between the $\mathrm{DB}$ and $\mathrm{CH}$ indices using the two clustering methods. Based on the $\mathrm{CH}$ index, FCMGWO performs better than FCM for images 3 and 4, but FCM is better than FCMGWO for images 1 and 2. The $\mathrm{CH}$ index also shows that FCMGWO is better than FCM in images with less detail. However, the DB indices of the two methods are almost identical with no difference in image type. Lack of ground truth was our main limitation in this study. 
Therefore, it is not possible to compare clustering results with indices such as sensitivity and specificity.

In future studies, the algorithm can be tested on other images with similar color intensity. Furthermore, the overall performance of the proposed method in images with more detail can be improved by a fuzzy algorithm modified via adding a more powerful objective function.

\section{Conclusions}

The results show that the FCMGWO method performs better on images with less detail than those with more detail. The hybrid algorithm distinguishes the cells better in images with less detail than in images with high detail. However, FCM exhibits unacceptable performance in both low- and highdetail images.

\section{Methods}

The image analysis consists of preprocessing and segmentation steps, which will be discussed in detail in subsequent sections.

\section{Image segmentation steps}

1. Slide image acquisition

2. Reducing the size of the image to $800 * 600$

3. Reducing the noise of the images using a median filter of size 5

4. Highlighting the cell nucleus via morphological closing

5. Using FCM clustering algorithms or the hybrid FCM clustering and gray wolf algorithm

\section{Actual images}

Imprint touch breast cytology images were utilized to examine the segmentation methods. All the images were confirmed by a pathologist. The images were produced with a magnification of 400x. 
The image for digital analysis was generated by a echoLAB camera mounted atop an echoLAB microscope. They were first resized to $800 * 600$ to reduce the processing time. All the analyses were performed using Python 3.8 and SPSS 26.

\section{Image preprocessing}

Image noise is the random change in the brightness or color data of an image(7) and can severely deteriorate image quality(8). In addition to denoising, preserving the edges and details of an image plays a key role in image processing(8). In this study, a median filter of size 5 has been used to reduce the noise from the camera. After using the median filter, morphological closing was employed to highlight the nucleus of the cell in the images.

\section{Image segmentation}

After preprocessing, image segmentation was performed via clustering techniques. Classification of tissue as malignant or benign requires detecting the nucleus in the cytology images. This is a challenging task since the images usually contain overlapping and clustered objects. In this study, FCM and FCMGWO clustering were used for image segmentation.

\section{FCM clustering}

FCM is a powerful unsupervised method for data analysis. This technique has been frequently used in image segmentation(9). FCM aims to divide the data inside the subspaces according to the distance criterion(5). The objects at the boundaries between different classes do not have to belong fully to one class but are rather assigned membership degrees between 0 and 1(9). FCM clustering was introduced by Bezdek in 1973. The objective function of FCM is defined as follows(10): 


$$
J_{m}=\sum_{i=1}^{n} \sum_{j=1}^{c} u_{i j}^{m}\left\|x_{i}-c_{j}\right\|^{2}
$$

where $m$ represents the degree of fuzziness and is a real number greater than $1, \mathrm{u}_{\mathrm{ij}}$ is the membership degree of the $\mathrm{i}^{\text {th }}$ datum in the $\mathrm{j}^{\text {th }}$ cluster, $\mathrm{x}_{\mathrm{i}}$ denotes the data points, and $\mathrm{c}_{\mathrm{j}}$ is the cluster center. Also, $\|\cdot\|$ represents the Euclidean distance, $\mathrm{n}$ is the number of data points, and $\mathrm{c}$ denotes the number of clusters(11).

The initial parameters were initialized as follows: Number of clusters=3; Fuzziness factor=1.5; Number of iterations $=5$.

\section{FCM implementation steps}

Step 1: Initialization of the number of clusters, $\mathrm{m}$, and $\mathrm{T}$ (maximum number of iterations) and random initialization of $u_{i j}$ for assigning Datum i to Cluster $j$.

Step 2: Determination of the cluster center using the following equation:

$$
C_{j}=\frac{\sum_{i=1}^{n} u_{i j}^{m} x_{i}}{\sum_{i=1}^{n} u_{i j}^{m}}
$$

Step 3: Determination of the change in the membership function matrix using the following equation:

Step 4: Calculation of J (u, v)

$$
u_{i j}=\frac{1}{\sum_{k=1}^{c}\left[\frac{d_{i j}}{d_{i k}}\right]^{\frac{2}{m-1}}}
$$

Step 5: Examination of the stop criterion (if $\left|\mathrm{J}_{\mathrm{t}+1}-\mathrm{J}_{\mathrm{t}}\right|<$ or $\mathrm{t}>\mathrm{T}$, the algorithm stops; otherwise it returns to Step 2.)

\section{Gray wolf optimization}

Optimization is a common method in machine learning for searching for the best solution or a sufficiently good solution. The GWO is a heuristic swarm intelligence optimization algorithm introduced by Mirjalili et al. in 2014(6). The best, second-best, and third-best responses are recorded as alpha, beta, and delta, respectively, and the rest of the wolves are considered as omega(12). 
Optimization algorithms require exploration and exploitation in a search space. In GWO, exploration refers to when the wolf leaves the initial search path in a specific context and turns to a new direction(12). Exploitation refers to when the wolf searches more accurately in the initial search path in a specific context(12). An optimization algorithm requires a good agreement between the exploration and exploitation steps for successful implementation(13). GWO has a high exploration capability in finding the best solution for the problem. This capability prevents the entrapment of the algorithm in local optima(6).

\section{Mathematical modeling of GWO}

\section{Encircling the prey}

Gray wolves encircle the prey during hunting. The following equations have been proposed to model the encirclement behavior(6):

$$
\begin{gathered}
\vec{D}=\left|\vec{C} * \vec{X}_{p}(t)-\vec{X}(t)\right| \\
\vec{X}(t+1)=\vec{X}_{p}(t)-\vec{A} * \vec{D}
\end{gathered}
$$

Where

$$
\begin{gathered}
\vec{A}=2 \vec{a} * \vec{r}_{1}-\vec{a} \\
\vec{C}=2 * \vec{r}_{2}
\end{gathered}
$$

where $\mathrm{t}$ is the current number of iterations, $\vec{A}$ and $\vec{C}$ are the coefficient vectors, $\vec{X}_{p}$ is the position vector of the prey, and $\vec{X}$ is the position vector of the gray wolf(6). D is the distance between the 
positions of the prey and the wolf at time t. The components $\vec{a}$ decrease linearly from 2 to 0 during the iteration, and $r_{1}$ and $r_{2}$ are random vectors in the range $[0,1](6)$.

\section{Prey}

Gray wolves are able to identify the hunting and encirclement locations. However, there is no idea of the optimal position of the prey in a search space(6). It is assumed that the alpha, beta, and delta are more knowledgeable about the potential position of the prey(6). Therefore, the first three obtained solutions are stored, and the other agents are responsible for updating their positions according to that of the best search agent(6). The following formulae have been presented in this regard(6):

$$
\vec{D}_{\alpha}=\left|\vec{C}_{1} \cdot \vec{X}_{\alpha}-\vec{X}\right|, \vec{D}_{\beta}=\left|\vec{C}_{2} \cdot \vec{X}_{\beta}-\vec{X}\right|, \vec{D}_{\delta}=\left|\vec{C}_{3} \cdot \vec{X}_{\delta}-\vec{X}\right|
$$

In updating, a hypothetical position must be considered for the prey since the position of the prey is unknown. The best option for this hypothetical position is the best position the wolves have been at so far. The position $\vec{X}_{p}(t)$ must be replaced by those of the alpha, beta, and delta wolves, and $\mathrm{D}_{\alpha}, \mathrm{D}_{\beta}$, and $\mathrm{D}_{\delta}$ must be calculated as the distances between these wolves and the prey, respectively.

$$
\begin{gathered}
\vec{X}_{1}=\vec{X}_{\alpha}-\vec{A}_{1} \cdot\left(\vec{D}_{\alpha}\right), \vec{X}_{2}=\vec{X}_{\beta}-\vec{A}_{2} \cdot\left(\vec{D}_{\beta}\right), \vec{X}_{3}=\vec{X}_{\delta}-\vec{A}_{3} \cdot\left(\vec{D}_{\delta}\right) \\
\vec{X}(t+1)=\frac{\vec{X}_{1}+\vec{X}_{2}+\vec{X}_{3}}{3}
\end{gathered}
$$

\section{GWO pseudo code}

Initialize the gray wolf positions $\mathrm{X}_{\mathrm{i}}(\mathrm{i}=1,2, \ldots, \mathrm{n})$

Initialize $\alpha, \mathrm{A}$, and $\mathrm{C}$

Calculate the fitness of every search agent

$\mathrm{X}_{\alpha}=$ the best search agent

$\mathrm{X}_{\beta}=$ the second-best search agent

$\mathrm{X}_{\delta}=$ the third-best search agent

$(\mathrm{t}<\mathrm{max}$ number of iterations) while

For each search agent

Update the position of the current search agent 
End for loop

Update $\alpha, \mathrm{A}$, and $\mathrm{C}$

Calculate the fitness of every search agent

Update $\mathrm{X}_{\alpha}, \mathrm{X}_{\beta}$, and $\mathrm{X}_{\delta}$

$\mathrm{t}=\mathrm{t}+1$

End of while

Return $\mathrm{X}_{\alpha}$

\section{Proposed method}

The proposed segmentation method is a technique that combines the benefits of FCM and GWO. FCM is a recursive algorithm that aims to find the cluster centers such that the dissimilarity function is minimized. The FCM-GWO method has been employed for the segmentation of images in order to counterbalance the drawbacks of FCM clustering. The cluster centers obtained from FCM are input to the GWO algorithm as initial positions to improve the FCM results and better distinguish the cell nucleus. The details of this hybrid technique are expressed in the following algorithm.

The parameters were initialized as follows: Number of clusters $=3$; Number of search agents $=5$; Fuzziness factor=1.5; Number of iterations $=5$; Lower bound of the search space $=0$; Upper bound of the search space $=225$.

\section{FCMGWO hybrid algorithm pseudo code}

Step 1: Read the cytology image

Step 2: Use a median filter for noise reduction

Step 3: Use morphological closing to highlight the cell nucleus

Step 4: Initialize the FCM parameters

Step 5: Obtain the cluster centers using FCM

Step 6: Initialize the gray wolf positions randomly; initialize the number of search agents and the maximum number of iterations

Step 7: Initialize $\alpha, \beta$, and $\delta$

Step 8: Assign the cluster centers obtained from FCM to the gray wolf positions

Step 9: Initialize the gray wolf positions $\mathrm{P}_{\alpha}, \mathrm{P}_{\beta}$, and $\mathrm{P}_{\delta}$

$\mathrm{t}=\mathrm{t}+1$

While $\mathrm{t}<$ Max-iteration

For each search agent 
Update the position of the current search agent

Compute the fitness function for each search agent

End For

Update $\mathrm{P}_{\alpha}, \mathrm{P}_{\beta}$, and $\mathrm{P}_{\delta}$

$\mathrm{t}=\mathrm{t}+1$

End while

Determine the best solution

\section{Validation of the clustering methods}

Two groups of indices were introduced to evaluate the clustering algorithms. The first group uses only the membership values $\left(\mathrm{u}_{\mathrm{ij}}\right)$, while the second group uses the membership matrix and the data(14). The partition coefficient (PC) and the partition entropy (PE) coefficient variance indices were selected from the first group, and the Calinski-Harabasz (CH) and Davies-Bouldin (DB) indices were chosen from the second group(14).

\section{Validity indices}

$\mathrm{V}_{\mathrm{PC}}$ and $\mathrm{V}_{\mathrm{pe}}$ are in the range [0,1], and optimal clustering has been obtained when $\mathrm{V}_{\mathrm{PC}}$ is maximum or $\mathrm{V}_{\mathrm{pe}}$ is minimum. DB index is in the range $(0, \infty)$. A lower value represents better clustering. A higher $\mathrm{CH}$ value represents better clustering.

\section{List of abbreviations}

FCM: Fuzzy c-means

GWO: Gray wolf optimization

CH: Calinski-Harabasz

DB: Davies-Bouldin

$V_{\text {pe: }}$ the partition entropy coefficient variance 
$\mathrm{V}_{\mathrm{pc}}$ : partition coefficient

\section{Declarations}

Ethics approval and consent to participate

Not applicable.

Consent for publication

Not applicable.

Availability of data and material

Not applicable.

Competing interests

We declare that we have no competing interest.

Funding

This study (IR.UMSHA.REC.1399.338) was approved by the Vice-Chancellor of Research and Technology of Hamadan University of Medical Sciences. The funders had no role in study design, data collection, and analysis, decision to publish, or preparation of the manuscript.

Authors' contributions

Conceptualization: M.M.KH, A.R.S, M.F; Data curation: M.M.KH, A.D; Formal analysis:

M.M.KH, A.R.S; Methodology: M.M.KH, A.R.S, M.F; Writing - original draft: M.M.KH, A.R.S;

Writing - review \& editing: M.M.KH, A.R.S; All authors read and approved the final manuscript.

Acknowledgements 
We would like to thank the staff of the pathology department of Besat Hospital in Hamadan and staff of the dissection laboratory of Hamadan University of Medical Sciences. Moreover, we thank the Vice-Chancellor of Research and Technology of Hamadan University of Medical Sciences (Construct No. 9904102207).

\section{References}

1. Dhanachandra N, Manglem K, JinaChanu Y. Image Segmentation Using K -means Clustering Algorithm and Subtractive Clustering Algorithm. Procedia Computer Science 2015;54:764-71.

2. Chen J, Shao H, Hu C. Image Segmentation Based on Mathematical Morphological Operator. In: Travieso-Gonzalez C, editor. Colorimetry and Image Processing: IntechOpen; 2018. p. 23-41.

3. Singh $\mathrm{TI}$, Laishram R, Roy S. Image segmentation using spatial fuzzy $\mathrm{C}$ means clustering and grey wolf optimizer. 2016 IEEE International Conference on Computational Intelligence and Computing Research (ICCIC) 2016:1-5.

4. Zanaty E. Determining the number of clusters for kernelized fuzzy C-means algorithms for automatic medical image segmentation. Egyptian Informatics Journal. 2012;13(1):39-58.

5. Benaichouche A, Oulhadj H, Siarry P. Improved spatial fuzzy c-means clustering for image segmentation using PSO initialization, Mahalanobis distance and post-segmentation correction. Digital Signal Processing. 2013;23(5):1390-400.

6. Mirjalili S, Mirjalili SM, Lewis A. Grey Wolf Optimizer. Advances in Engineering Software. 2014;69:4661.

7. Sayed G, Hassanien A, Schaefer G. An automated computer-aided diagnosis system for abdominal CT liver images. Procedia Computer Science. 2016;90:68-73.

8. Sakthivel N, Prabhu L. Mean-Median Filtering For Impulsive Noise Removal. International Journal of Basic and Applied Science. 2014;2(4):47-57.

9. Ravindraiah R, Tejaswini K. A survey of image segmentation algorithms based on fuzzy clustering. International Journal of Computer Science and Mobile Computing. 2013;2(7):200-6.

10. Ma L, Li Y, Fan S, Fan R. A hybrid method for image segmentation based on artificial fish swarm algorithm and fuzzy-means clustering. Computational and mathematical methods in medicine. $2015 ; 201.5$

11. Tongbram S, Shimray B, Singh L, Dhanachandra N. A novel image segmentation approach using fcm and whale optimization algorithm. Journal of Ambient Intelligence and Humanized Computing. 2021:1-5.

12. Wang J-S, Li S-X. An Improved Grey Wolf Optimizer Based on Differential Evolution and Elimination Mechanism. Scientific reports. 2019;9(1):7181.

13. Črepinšek M, Liu S, Mernik M. Exploration and exploitation in evolutionary algorithms: A survey. ACM computing surveys. 2013;45(3):1-33.

14. El-Melegy $M$, Zanaty EA, Abd-Elhafiez WM, Farag A. on cluster validity indices in fuzzy and hard clustering algorithms for image segmentation. IEEE International Conference on Image Processing, ICIP. 2007;6:5-8. 


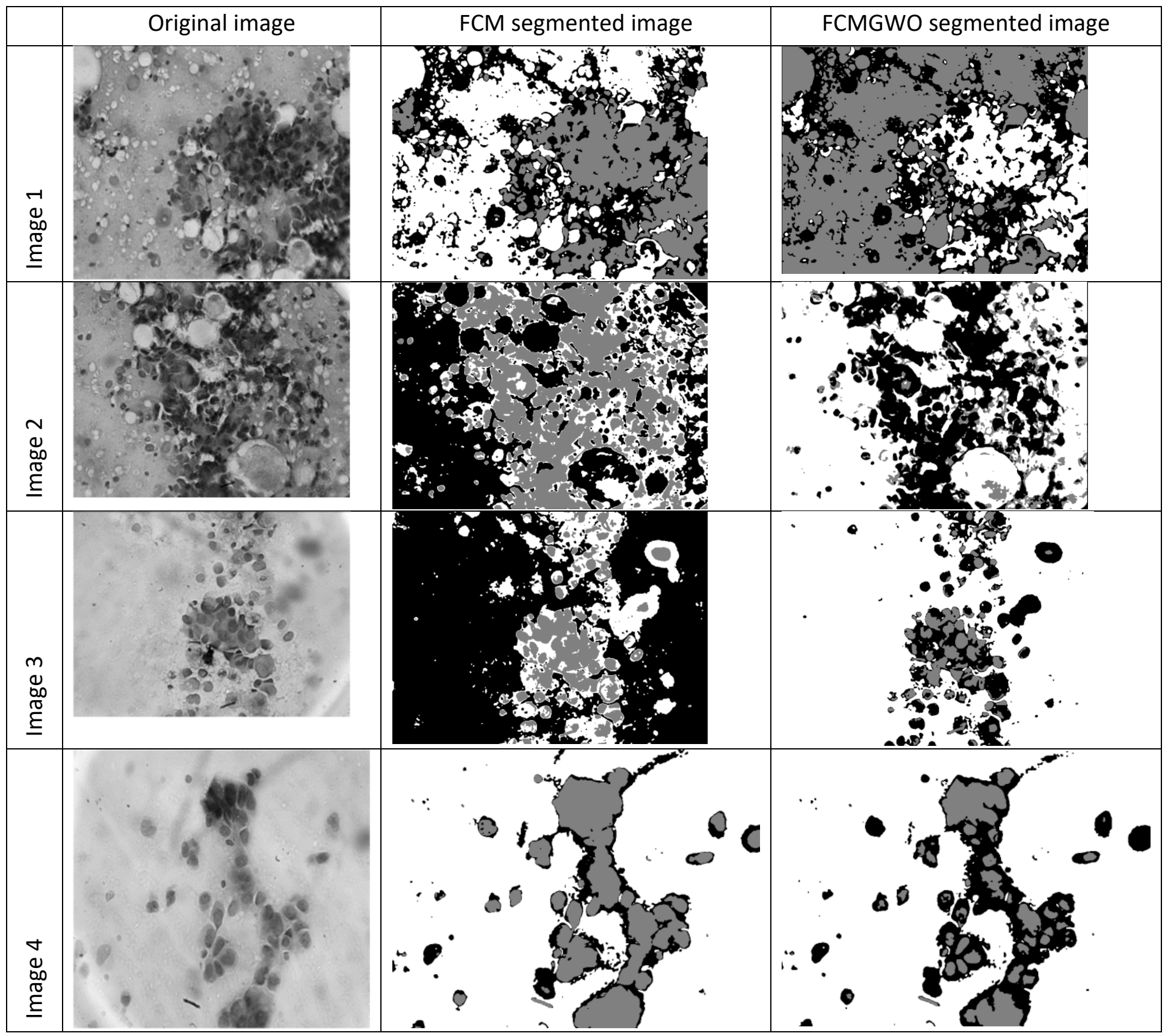

Figure 1. Cytology images based on original and segmented using FCM and FCMGWO methods. The original images are samples of the frozen section of Breast cancer from a 50-year-old woman. The diagnostic result is invasive ductal carcinoma with a score of 7.9 and grade II/III. The tumor size is $5.5 \times 5 \times 3$. The original images were first resized to $800 \times 600$ pixels. 


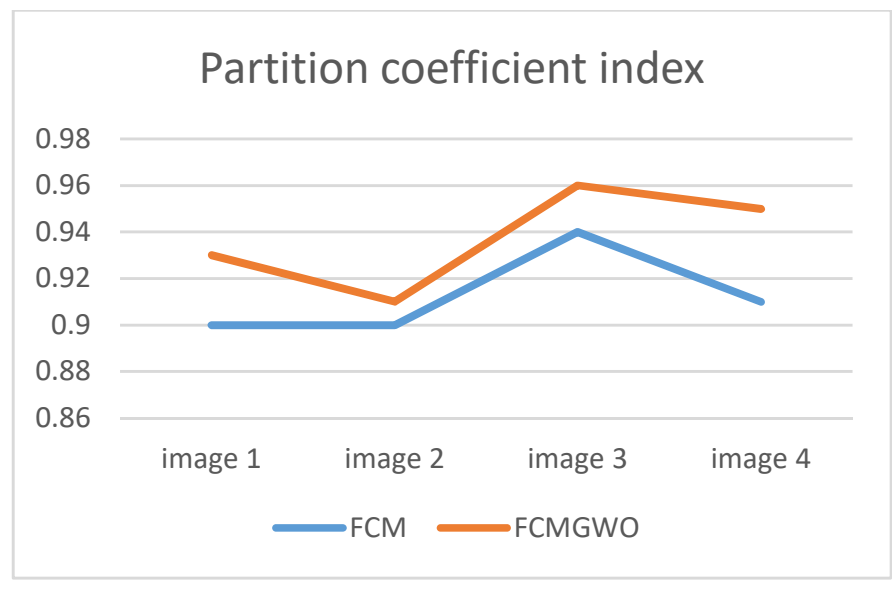

a

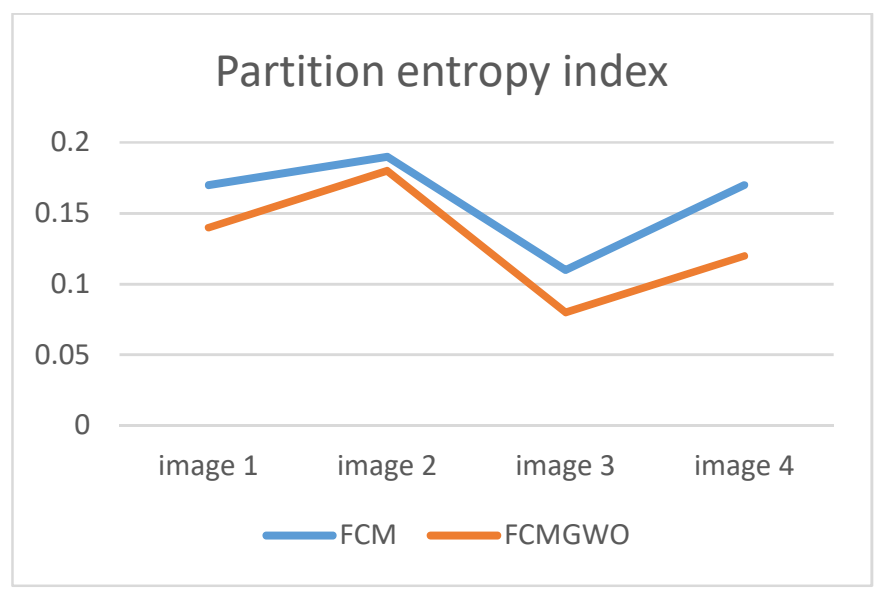

b

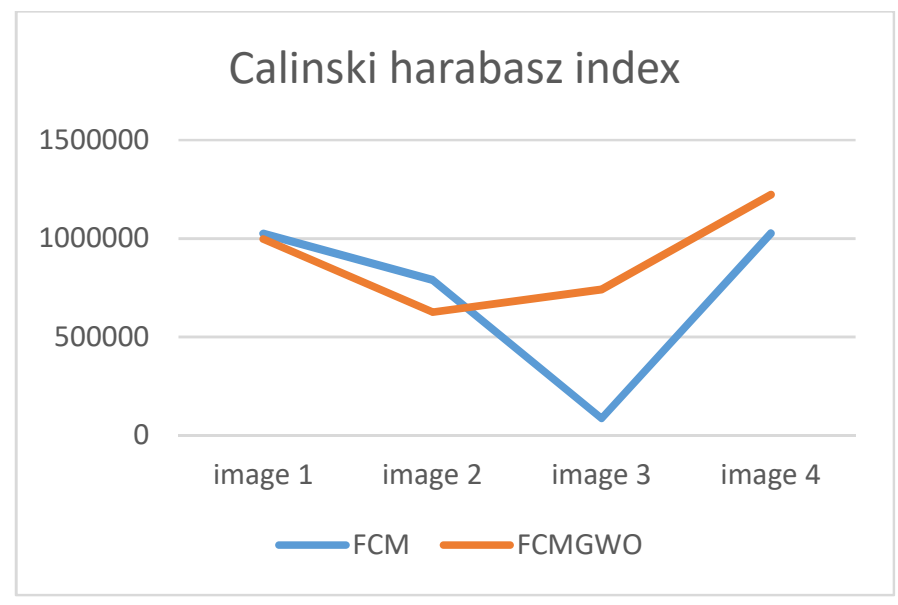




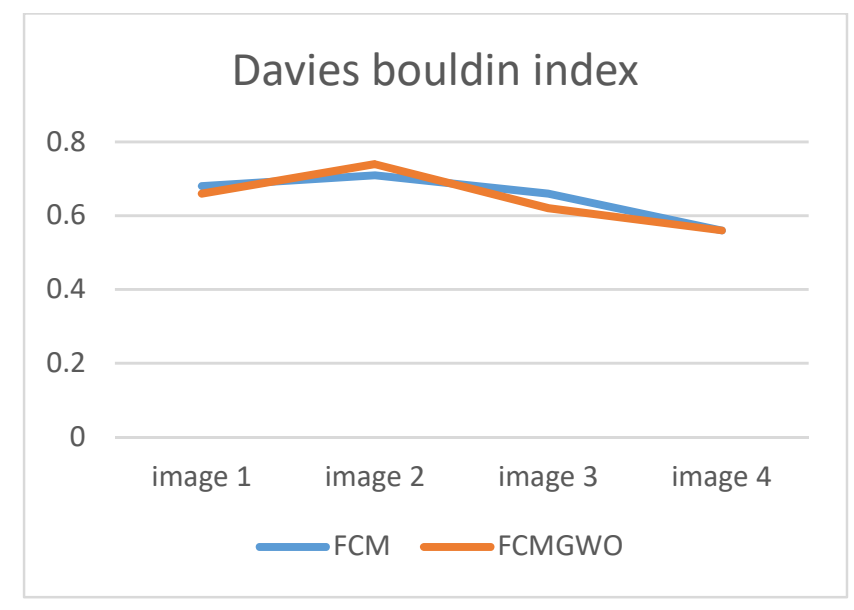

d

Figure 2. Validation indexes for comparing FCM and FCMGWO. The FCM and FCMGWO methods have a significant difference with respect to the Vpc and Vpe indices. However, there is no significant difference between the performances of the two clustering methods with respect to the Calinski-Harabasz and Davies-Bouldin indices.

Table 1. Paired t-test results indicating the difference between the performance of the indices

\begin{tabular}{|c|c|c|c|}
\hline index & Methods & $\mathrm{t}$ & $\mathrm{p}$-value \\
\hline $\mathrm{V}_{\mathrm{pc}}$ & $\mathrm{FCM}:$ FCMGWO & -3.873 & 0.030 \\
\hline $\mathrm{V}_{\mathrm{pe}}$ & FCM:FCMGWO & 3.674 & 0.035 \\
\hline $\mathrm{DB}$ & FCM:FCMGWO & 0.502 & 0.650 \\
\hline $\mathrm{CH}$ & FCM:FCMGWO & -0.920 & 0.426 \\
\hline
\end{tabular}




\section{Supplementary Files}

This is a list of supplementary files associated with this preprint. Click to download.

- Supplementary.docx 